\title{
SUPPLEMENT
}

\author{
Berin T. Demir ${ }^{1}$ \\ Nezibe A. Bayram ${ }^{2}$ \\ Zübeyde Ayturk ${ }^{3}$ \\ Hüsamettin Erdamar ${ }^{2}$ \\ Pelin Seven ${ }^{4}$ \\ Aysegül Calp 4 \\ Merve Sazak ${ }^{4}$ \\ Hatice G. Ceylan ${ }^{4}$
}

${ }^{1}$ Turgut Özal University, Faculty of Medicine, Department of Anatomy, Ankara, Turkey

${ }^{2}$ Turgut Özal University, Faculty of Medicine, Department of Biochemstry, Ankara, Turkey

3Turgut Özal University, Faculty of Medicine, Department of Neurology, Ankara, Turkey

${ }^{4}$ Turgut Özal University, Faculty of Medicine, Ankara, Turkey

\section{Structural Changes in the Cerebrum, Cerebellum and Corpus Callosum in Migraine Patients}

\begin{abstract}
Purpose: The purpose of this study was to demonstrate the relationship among the cerebrum, cerebellum and corpus callosum in migraine patients.

Methods: This work was conducted with cooperation of the Turgut Özal Medical Faculty, Department of Anatomy and Neurology. Migraine patients were divided into four groups: new patients; $1-5$ years; 5-10 years; and, more than 10 years. All patients $(n=75)$ and control subjects $(\mathrm{n}=20)$ underwent Magnetic Resonance Imaging (MRI) and brain images were processed by ONIS and Image J. Data were analyzed using the planimetric method.

Results: Cerebrum, cerebellum and corpus callosum volume were calculated for all subjects. The footprints of the callosum were as follows: healthy control subjects, new patients and 1-year patients: $12.8 \%, 5$ years: $11.7 \%$ and more than 10 years: $10.7 \%$. The cerebrum volume was as follows: healthy control subjects: $1152 \mathrm{~cm}^{3}, 5-10$ years: $1102 \mathrm{~cm}^{3}$ and more than 10 years: $1002 \mathrm{~cm}^{3}$.
\end{abstract}

Discussion: The results of our study showed atrophy in the cerebrum, cerebellum and corpus callosum of chronic migraine patients. This atrophy was greater in the patients with aura migraines.

Conclusion: Our study confirmed that a migraine is an episodic disease that seriously affects the CNS.

\section{Correspondence to:}

Berrin Tuğtağ Demir, Turgut Özal University,

Faculty of Medicine, Department of Anatomy, Ankara, Turkey

E-mail: berrintugtag@hotmail.com 
A migraine is a neurological disorder; defined as primarily paroxysmal attacks and autonomic nervous system dysfunction and is often characterized by one-sided head pain $[1,2]$. Although migraine is common and easily diagnosed, its etiology is unresolved. This leads to difficult to diagnose because the show before a headache itself in many neurological diseases.-Migraine is seen in $10-15 \%$ of the overall adult population, and is more prevalent in women [1], and children [3]. It leads to serious social problems for individuals. Clinical studies have shown that individuals with migraine, a decrease in the volume of cortical and subcortical structures had occurred. In addition, there was clinical evidence of lesions in white matter and cerebellum in migraine patients $[4,5]$. In neuropsychological and neurophysiological diseases, the corpus callosum, which connects the two hemispheres, is the first part of the brain affected by a migraine, and has a critical role on the basis of this disease but it is not clearly understood how it is affected $[6,7]$.

There are no published studies on the corpus callosum that directly address the connection between the motor and cognitive functions. We intended to clarify this, as well as the relationship among the cerebrum, cerebellum and corpus callosum volumes and the effect of the disease duration on these volumes. We also report, for the first time, the use of this MRI method for this patient group.

\section{Patients and Methods}

\section{Patients}

This study was approved by Turgut Ozal University Medical Faculty institutional review board and the ethics committee. Written informed consent was obtained from all participants at inclusion, in accordance with the Helsinki Declaration.

We studied, prospectively, 95 (32 male and 63 female) healthy control subjects $(n=20)$ and migraine patients $(n=75)$ who had been admitted to the Clinic of Turgut Ozal University Hospital Ankara, Turkey. Migraine diagnosis was based on the International Classification of Headache Disorders diagnostic criteria. Criteria based on healthy individuals and individuals with a diagnosis of a migraine were made on brain magnetic resonance (MR) images. Classification with aura or without aura was based on International Classification of Headache Disorders diagnostic criteria among migraine patients.

\section{Study Design}

The patients were divided into four groups, according to the duration of illness:
Group 1: Healthy individuals; people without any a headache or neurodegenerative diseases $(n=20)$;

Group 2: New patients; less than 1 year after diagnosis with a migraine $(\mathrm{n}=17)$;

Group 3: Patients diagnosed with a migraine between 1-5 years $(n=20)$;

Group 4: Patients diagnosed with a migraine between 5-10 years $(n=20)$; and,

Group 5: Patients diagnosed with a migraine more than 10 years $(n=18)$.

\section{Projection Area Length (PAL) Value for Corpus Callosum}

The cross-sectional surface area measured by means of the planimetry method using Dicom Works (Version 1.3.5. France. http://dicom.online.fr.) and ImageJ (ImageJ, $1.37 \mathrm{v}$ : http://rsb.info.nih.gov/ij/). Digitally imported movies or pictures calculated using the following formula:

$$
\mathrm{PAL}=\frac{A}{l^{2}}
$$

where PAL is the projected area length, $\mathrm{A}$ is structure or body of the projection area of interest and $\mathrm{L}$ is the measured reference length. Results are in the form of a percentage.

In this study, the section in which CC looks the best was chosen from among 18 consecutive sections taken from MR images in the sagittal plane; usually, the 9th and 10th sections. ONIS name designated sections before transferring the data to the digital environment Image $\mathrm{J}^{\odot}$ program and then displayed the boundaries were drawn in CC.

\section{Volume Calculation for Cerebrum and Cerebellum}

The coefficient error of planimetric volume estimations was calculated using the following formula described in previous studies [8-10]:

$C E=\left(\sum_{i=1}^{m} A_{i}\right)^{-1} \times\left[\frac{1}{12} \times\left(3 \sum_{i=1}^{m} A_{i}^{2}+\sum_{i=1}^{m-2} A_{i} \times A_{i+2}-4 \sum_{i=1}^{m-1} A_{i} \times A_{i+1}\right)\right]^{1 / 2}$

A study reported [8], using hand-hold mouse, the rater traced around the area of interest within the slice. Calculation of cerebrum volume, coefficient error of estimates and other data were done using Microsoft Excel. 
TABLE 1. Statistical results of comparison between corpus callosum, cerebellum, and cerebrum volume

\begin{tabular}{|c|c|c|c|c|}
\hline & Statistical Method & Corpus Callosum & Cerebellum & Cerebrum \\
\hline \multirow{2}{*}{ Corpus callosum } & Pearson Correlation & 1 & $.304^{* *}$ & $.283^{*}$ \\
\hline & Sig. (2-tailed) & & .009 & .016 \\
\hline \multirow{2}{*}{ Cerebellum } & Pearson Correlation & $.304^{* *}$ & 1 & .177 \\
\hline & Sig. (2-tailed) & .009 & & .136 \\
\hline \multirow{2}{*}{ Cerebrum } & Pearson Correlation & $.283^{*}$ & .177 & 1 \\
\hline & Sig. (2-tailed) & .016 & .136 & \\
\hline \multirow{2}{*}{$\begin{array}{l}\text { Duration of the } \\
\text { migraine }\end{array}$} & Pearson Correlation & $-.435^{* *}$ & $-.359^{* *}$ & -.192 \\
\hline & Sig. (2-tailed) & .000 & .002 & .107 \\
\hline
\end{tabular}

${ }^{*}$ and ${ }^{* *}$ mean $\mathrm{p}<0.05$

\section{Statistical Analysis}

The SPSS statistical software package (SPSS, version 20 for windows; SPSS Inc., Chicago, Illinois, USA) was used to perform all statistical calculations. The results were presented as the standard error of the mean. Independent samples of $\mathrm{t}$-tests were used to compare the mean values of the groups (migration-control). The non-numeric comparisons (e.g., duration of migraine) were evaluated using an ANOVA.

\section{Results}

The youngest patients were 18 years old, the eldest was 61 years old and the average of the age was $40.5 \pm 12.9$ years.

The area covered by the corpus callosum in the brains of healthy subjects and migraine patients was different among the five groups: Group 1 was 13.2\%; Group 2 was 13.1\%; Group 3 was $12.6 \%$; Group 4 was $11.9 \%$; and, Group 5 was $10 \%$. The volume of the cerebrum was also different: Group 1 was $1152.1 \mathrm{~cm}^{3}$; Group 2 was $112.7 \mathrm{~cm}^{3}$; Group 3 was $110.4 \mathrm{~cm}^{3}$; Group 4 was $1086 \mathrm{~cm}^{3}$; and, Group 5 was $1002.5 \mathrm{~cm}^{3}$. The volume of the cerebellum was also different: Group 1 was $114.9 \mathrm{~cm}^{3}$; Group 2 was $114 \mathrm{~cm}^{3}$; Group 3 was $110 \mathrm{~cm}^{3}$; Group 4 was $106.7 \mathrm{~cm}^{3}$; and, Group 5 was $101.4 \mathrm{~cm}^{3}$.

The average value of the CC PAL in healthy subjects was $13.2 \pm 3.2 \%$, the volume of cerebrum was $1152.1 \pm 150 \mathrm{~cm}^{3}$ and the volume of the cerebellum was $114.4 \pm 9.3 \mathrm{~cm}^{3}$. For patients with a migraine, the average value of CC PAL was $12 \pm 2.4 \%$, the cerebrum volume was $1079.4 \pm 200 \mathrm{~cm}^{3}$ and the cerebellum volume was $108.1 \pm 9 \mathrm{~cm}^{3}$. Figure 1 summarizes the results for corpus callosum, cerebrum and cerebellum.
In our study, we divided patients with a migraine into two groups according to with aura or without aura. In migraine patients with aura, the volume of corpus callosum and cerebellum was decreased but it was not significant ( $p>0.05$ ).

According to the Pearson correlation test, according to the cerebrum with and there was a positive correlation between the corpus callosum, the cerebellum, and cerebrum.

The size of the corpus callosum was increasing while the cerebrum was expanding or getting smaller. There was a negative correlation between migraine disease duration, cerebellum, and cerebrum. This means when the disease duration decreased, corpus callosum space occupying in the brain (PAL) was significantly decreasing $(\mathrm{p}=0.00)$ and the volume of the cerebellum was reducing $(p=0.02)$. Statistical results are shown in Table 1.

\section{Discussion}

We performed this work to determine if there were structural and volume change in the cerebrum, cerebellum and corpus callosum in patients with more severe migraine with aura and to determine if a decrease in brain volume correlated with increasing disease duration.

Our results indicated that a decreased volume of cerebellum correlate with increased migraine duration, and this decreased cerebellum volume was statistically significant at longer than five years migraine patients. Bilgiç et al. (2015) reported a significant reduction in the infratentorial region and the volume of cerebellum with migraine patients, but it is not known whether there were 


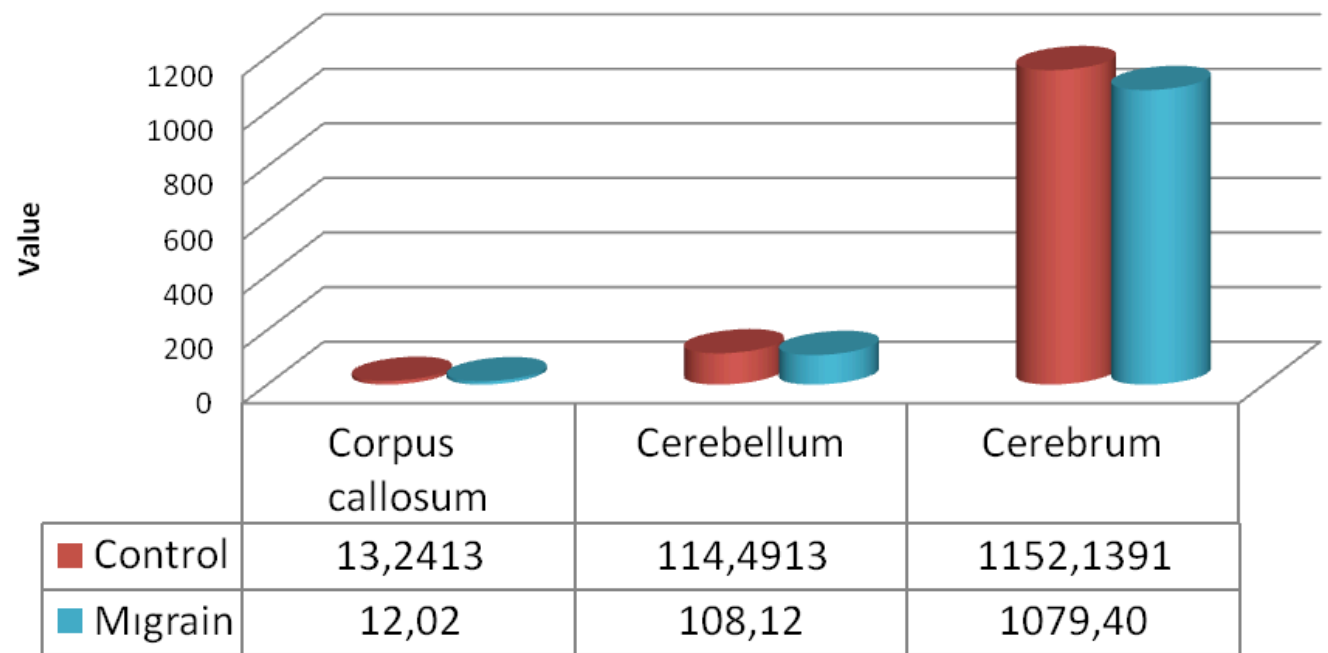

FIGURE 1. Comparison of the corpus callosum, cerebellum and cerebrum volume between healthy individuals (controls) and patients with a migraine.

structural changes [11]. This relationship between a migraine and decrease cerebellum volume leads to cognitive and motor function loss over time. Zielman $e t$ al. stated that a migraine caused atrophy in the brain and led to cerebellar neural damage [12]. In migraine patients, vestibulocochlear dysfunction and balance problems often arise because of neuronal loss in the cerebellum [13]. In addition, problems occur with the perception of pain as the cerebellum plays a role in the pathogenesis of pain [14].

Women make up the majority of our study population because migraines are seen more in women than in men. We found that the corpus callosum and cerebellum of female patients are more affected by a migraine than those of male patients. Hormonal changes in women may affect mood and result in increased susceptibility to depression, so women with a migraine are more at risk [15]. Studies have claimed that there are neuronal losses in the frontal and temporal lobes, cingulate gyrus, parietal cortex, white matter of the occipital cortex and brain stem contains cranial fossa posterior structures $[15,16]$. Bilgiç et al. also reported that the extent of the decrease in the cerebrum depends on the extent of reduction in the gray matter. In contrast, other studies have reported thickening of gray matter, especially in the somatosensory and visual cortexes [17]. We did not find a statistically significant reduction in the cerebellum volume, although this may be due to the low number of patients. Liu et al. did not find a relationship between headaches and brain gray matter volume, and they pointed out there were inconsistencies between the headache activities and structural changes of the brain [16]. In our study, we saw a volumetric difference between the brains of patients with migraines with an aura and migraine without an aura, although the difference was not statistically significant.

The planimeter method, which we used in this study, has not been used for similar research on migraine patients previously. We were able to get an idea of the space that the structures occupy in the brain by reducing the error coefficient minimum using planimetry, which is stereology technique.

It was quite difficult to obtain accurate data on the size of the corpus callosum as it is a fibrous structure. The corpus callosum is also an indicator of neurological disease at every stage of life. It is vitally important to identify disease, especially during old age, as specific cognitive function can be affected by not only by axonal 
loss but also by axonal damage [18]. Yuan et al. stated that there was a thinning, especially in the genu of corpus callosum, as a result of functional imaging techniques [19]; however, we were not able to get data regarding the size of regional corpus callosum with our MR images.

Corpus callosum atrophy was found to occur in many neuropsychological diseases, including Alzheimer's disease and depression, and it affected many structures including thalamic and basal ganglia [20]. We observed that the corpus callosum is statistically smaller in migraine patients compared with healthy individuals. Shrinkage was significantly associated with longer disease duration. This was particularly evident in the patients suffering from migraine over the course of 10 years, where up to $20 \%$ decrease in corpus callosum volume was seen the corpus callosum is simply shortened more in women than in men.

In conclusion, our study showed that atrophy occurred in both the cerebellum and corpus callosum of individuals with a migraine. This atrophy is especially marked in patients with long-term migraines and patients with migraines with an aura. Episodic migraine is a serious disease that affects the CNS and causes interhemispheric communication, visual disturbances, vestibular cochlear disorders, loss of function and a reduction in an individual's quality of life. With these changes there is a breakdown of the anatomical structure of cortical and subcortical elements; however, we could not evaluate structure-function relationships using our multimodal MRI techniques because of insufficient infrastructure. In subsequent studies, the underlying etiologic relationship between migraine duration and migraine attacks with brain functioning can be investigated.

\section{References}

1. Bashir A, Lipton RB, Ashina S, Ashina M. Migraine and structural changes in the brain. A systematic review and meta-analysis. American Academy of Neurology 2013; 81(1): 1260-1268.

2. Erdélyi-Bótor S, Aradi M, Olayinka Kamson D, Kovács N, Perlaki G, Orsi G, Anett Nagy S, Schwarcz A, Dóczi T, Komoly S, Deli G. Trauninger A, Pfund Z. Changes of Migraine-Related White Matter Hyper intensities After 3 Years: A Longitudinal MRI Study. Headache 2015; 55: 55-70.
3. Stovner, Hagen K, Jensen R, Katsarava Z, Lipton RB, Scher AI, Steiner TJ, Zwart JA. The global burden of headache: a documentation of headache prevalence and disability worldwide. Cephalalgia 2007; 27: 193-210.

4. Kruit MC, van Buchem MA, Launer LJ, et al. Migraine is associated with an increased risk of deep white matter lesions, subclinical posterior circulation infarcts and brain iron accumulation: The population-based MRI CAMERA study. Cephalalgia 2010; 30: 129-136.

5. Gudmundsson LS, Scher AI, Aspelund T, et al. Migraine with aura and risk of cardiovascular and all cause mortality in men and women: prospective cohort study. BMJ 2010; 341:c3966.

6. Kruit MC, van Buchem MA, Hofman PAM, Bakkers Jacobus TN, Terwindt GM, Ferrari MD, Launer LJ. Migraine as a Risk Factor for Subclinical Brain Lesions JAMA. 2004; 291(4): 427-434.

7. Yuan K, Qin W, Liu P, Zhao L, Yu D, Zhao L, Dong M, Liu J, Yang X, Deneen KMV, Liang F, Tian J. Reduced Fractional Anisotropy of Corpus Callosum Modulates Inter-Hemispheric Resting State Functional Connectivity in Migraine Patients without Aura. PLOS One 2012; 7: 9.

8. Acer N, Sahin B, Usanmaz M, Tatoglu H, Irmak Z. Comparison of point counting and planimetry methods for the assessment of cerebellar volume in human using magnetic resonance imaging: a stereological study. Surg Radiol Anat 2008: 30; 335-339.

9. Acer N, Sahin B, Bas O, Ertekin T, Usanmaz M. Comparison of three methods for the estimation of total intracranial volume: stereologic, planimetric, and anthropometric approaches. Ann Plast Surg 2007: 58; 48-53.

10. Acer N, Sahin B, Ucar T, Usanmaz M. Unbiased Estimation of the Eyeball Volume Using the Cavalieri Principle on Computed Tomography Images. The Journal of Craniofacial Surgery 2009; 20: 233-237.

11. Bilgic B, Kocaman G, Arslan AB, Noyan H, Sherifov R, Alkan A, Asil T, Parman B. Volumetric differences suggest involvement of cerebellum and brainstem in chronic migraine. Cephalalgia 2016; 36(4): 301-8.

12. Zielman R, Teeuwisse W, Bakels F, et al. Biochemical changes in the brain of hemiplegic migraine patients measured with 7 tesla 1H-MRS. Cephalalgia 2014; 34: 959-967.

13. Hougaard A. Investigations of functional and structural changes in migraine with aura by magnetic resonance imaging. Dan Med J 2015; 62(8): B5129.

14. Hubbard CS, Khan SA, Keaser ML, Mathur VA, Goyal M, Seminowicz D. Altered Brain Structure and Function Correlate with Disease Severity and Pain Catastrophizing in Migraine Patients. Neuro. 2014; e1(1): 1-17.

15. Gudmundsson LS, Sigurdsson S, Geerlings MI. Vidal JS, Gudny Eiriksdottir, Garcia MI, Harris TB, Kjartansson O, Aspelund T, Buchem MA, Gudnason V, Launer LJ. Migraine, depression, 
and brain volume The AGES-Reykjavik Study. Neurology 2013; 80: 2138-44.

16. Liu J, Lan L, Li G, Yan X, Nan J, Xiong S, Yin Q, Deneen KM, Gong Q, Liang F, Qin W, Tian J. Migraine-Related Gray Matter and White Matter Changes at a 1-Year Follow-Up Evaluation. The Journal of Pain 2013; 14(12): 1703-1708.

17. Maleki N, Balecca 1, Brown J, Mcewen B, Brustein R, Borsook D. Common hippocampal structural and functional changes in Migraine. Brain Struct Funct 2013; 218(4): 903-912.

18. Hou J, Pakkenberg B. Age-related degeneration of corpus callosum in the 90 years measured with stereology. Neurobiology of Aging 2012; 33: 1009.e1-1009.e9.

19. Yuan JY, Wang SK, Guo XJ, Hu WL. Acute infarct of the corpus callosum presenting as alien hand syndrome: evidence of diffusion weighted imaging and magnetic resonance angiography. BMC Neurology 2011; 11: 142.

20. Xu Qiang, Lu Wei, Qiu Xuan, Yang Shu, Li Chen, Zhang Wei, Tang Yong. Age related changes in the myelinated fibers of corpus callosum. Neuroscience Letters 2011; 499: 208- 212. 